Research Paper

\title{
Naringenin exerts anticancer effects by inducing tumor cell death and inhibiting angiogenesis in malignant melanoma
}

\author{
Jawun Choi, Dae-Hyo Lee, Hyuk Jang, Sang-Youel Park and Jae-Won Seol ${ }^{\bowtie}$ \\ College of Veterinary Medicine, Jeonbuk National University, Iksan, Jeollabuk-do 54596, Republic of Korea \\ $\triangle$ Corresponding author: College of Veterinary Medicine, Jeonbuk National University, Iksan, Jeollabuk-do 54596, Republic of Korea \\ (c) The author(s). This is an open access article distributed under the terms of the Creative Commons Attribution License (https://creativecommons.org/licenses/by/4.0/). \\ See http://ivyspring.com/terms for full terms and conditions.
}

Received: 2020.02.11; Accepted: 2020.10.01; Published: 2020.10.18

\begin{abstract}
Malignant melanoma is one of the most deadly skin cancer, due to its aggressive proliferation and metastasis. Naringenin, abundantly present in citrus fruits, has widely studied in cancer therapy. In this study, we investigated whether naringenin also has anticancer effects against B16F10 murine and SK-MEL-28 human melanoma cells. Moreover, we assessed the effects of naringenin treatment on angiogenesis of HUVECs and ex vivo sprouting of microvessels. Naringenin inhibited tumor cell proliferation and migration in a dose-dependent manner in B16F10 and SK-MEL-28 cells, which is supported by the results that phosphorylation of ERK1/2 and JNK MAPK decreased. Furthermore, naringenin induced cell apoptosis. Western blot analysisshowed naringenin treatment significantly upregulated the protein expression of activated cas3 and PARP in B16F10 and SK-MEL-28 cells. In addition, in vitro and ex vivo angiogenesis assays demonstrated that naringenin treatment potently suppressed EC migration, tube formation, and sprouting of microvessels. RT-PCR analysis showed that naringenin treatment significantly reduced the mRNA expression of Tie2, but did not inhibit the expression of Ang2. In conclusion, present study demonstrates the anticancer effects of naringenin by its induction of tumor cell death and inhibition of angiogenesis in malignant melanoma, suggesting that naringenin has potential as a safe and effective therapeutic agent to treat melanoma.
\end{abstract}

Key words: Flavonoids, Naringenin, Apoptosis, Angiogenesis, Melanoma, HUVECs

\section{Introduction}

Malignant melanoma is the most lethal skin cancer, accounting for $60 \%$ of skin cancer-related deaths [1]. The global incidence of melanoma has steadily continued to rise over the last two decades [2]. Diagnosis of melanoma at an early stage followed typically by surgical excision is associated with a favorable prognosis. However, melanoma has a peculiar tendency to metastasize to distant organs at an early stage of progression. Thus, additional treatment, such as chemotherapy, radiation, and immunotherapy, is often required after surgery [3]. In particular, although chemotherapy might be a suitable approach to treat metastatic melanoma, limitations of conventional chemotherapy include severe pain, adverse effects, and even recurrence [4]. Therefore, the discovery of new compounds that are safe and effective against melanoma is becoming increasingly important.

Inhibition of tumor-associated angiogenesis has become a promising strategy for cancer therapy during the last decade. Angiogenesis, new blood vessel formation from existing vessels, plays an important role in various diseases including cancer. Sufficient blood vessel supply is essential for tumor growth and progression, since it provides oxygen and nutrients, and removes cellular metabolites. In addition, the tumor vasculature is the major route of metastasis of cancer cells from the primary site to distant organs. Anti-angiogenic effects of a variety of phytochemicals have been demonstrated in various cancer cells in vitro as well as using in vivo tumor models [5-7]. The anti-angiogenic effect of gallic acid 
in ovarian cancer is due to the downregulation of the Phosphatase and tensin homologue deleted on chromosome 10/Protein kinase B/Hypoxia-inducible factor-1alpha (PTEN/AKT/HIF-1a) pathway and inhibition of vascular endothelial growth factor (VEGF) expression [8]. The combination of curcumin and (-)-epigallocatechin-3-gallate (EGCG) has anti-angiogenic activities in colorectal carcinoma by blocking the Janus kinase/Signal transducer and activator of transcription 3/Interleukin-8 (JAK/ STAT3/IL-8) pathway [9]. Given the importance of angiogenesis in cancer, identification of plant-derived compounds with anti-angiogenic activity against cancer cells and minimal toxicity to normal cells is an important research goal.

Flavonoids derived from plants have been actively investigated for cancer chemotherapy because of their therapeutic effects against various human cancers. Naringenin is one of the most important plant flavonoids. It is abundantly present in citrus fruits that include grapefruit, orange, and mandarin. Previous studies have demonstrated extensive pharmacological properties for naringenin, which include antioxidant, anti-diabetic, and anti-inflammatory [10]. Naringenin also has anticancer effects due to the induction of apoptosis and cell cycle arrest in a variety of cancer cell lines, including MDA-MB-231 breast tumor cells [11], HepG2 human hepatocellular carcinoma cells [12], E0771 mammary tumor cells [13], and PC3 and LNCaP prostate cancer cells [14]. A recent study suggested an important role for naringenin as an anti-angiogenic chemopreventive agent [15].

The present study was undertaken to assess the simultaneous anticancer and anti-angiogenic bioactivities of naringenin. The findings suggest that naringenin can be developed as a safe and effective drug to treat melanoma.

\section{Materials and Methods}

\section{Cell culture and reagents}

B16F10 murine and SK-MEL-28 human melanoma cell lineswere purchased from the Korean Cell Line Bank (Seoul, Korea). The cells were cultured in Dulbecco's modified Eagle's medium (B16F10 cells) or modified Eagle's medium (SK-MEL-29 cells, Gibco, Grand Island, NY, USA) supplemented with $10 \%$ fetal bovine serum (Atlas Biologicals, For Collins, Co, USA), $100 \mathrm{U} / \mathrm{ml}$ penicillin and $100 \mu \mathrm{g}$ streptomycin (Sigma-Aldrich). Human umbilical vein endothelial cells (HUVECs) and their growth medium (Endothelial Cell Growth Medium) were purchased from PromoCell GmbH (Heidelberg, Germany). The cells were used at passage $4-5$ in all experiments. All cells were incubated at $37{ }^{\circ} \mathrm{C}$ with $5 \% \quad \mathrm{CO}_{2}$. Naringenin was dissolved in dimethyl sulfoxide to obtain a $200 \mu \mathrm{M}$ stock solution, which was then diluted with media.

\section{Cell viability analysis}

Cell viability was determined by a 3-(4,5-dimethylthiazol-2-yl)-2,5-diphenyltetrazolium bromide (MTT) assay. B16F10 and SK-MEL-28 cells were seeded in 24 -well plates $\left(1 \times 10^{5}\right.$ cells/well $)$ overnight and treated with or without naringenin. After $24 \mathrm{~h}$, the media was carefully removed and 300 $\mu \mathrm{l}$ of MTT solution (0.5 mg MTT/ml medium) was added to each well, and plates were incubated for $2 \mathrm{~h}$ at $37^{\circ} \mathrm{C}$. The media was then replaced with $500 \mu \mathrm{l}$ of dimethyl sulfoxide, and the plates were shaken for 10 $\mathrm{min}$. Then, $200 \mu \mathrm{l}$ of the sample were transferred to a 96-well microplate. The results were quantified by measuring the absorbance at $570 \mathrm{~nm}$ with a microplate reader (Spectramax M2; Molecular Devices, CA, USA).

\section{Flow cytometry assays}

Cell apoptosis was assessed by the Annexin V assay (Santa Cruz Biotechnology, Inc., Dallas, TX, USA) according to the manufacturer's protocol using a flow cytometry method. Annexin V content was determined by measuring fluorescence at $488 \mathrm{~nm}$ (excitation) and $525 \mathrm{~nm}$ (emission) using a Guava easyCyteHT system (Millipore, Billerica, MA, USA).

\section{In vitro scratch migration assays}

Melanoma cell lines (B16F10 cells and SK-MEL-28 cells) and HUVECs were grown on 6-well plates. Confluent monolayer cells were scratched manually using a sterile $1000 \mu \mathrm{l}$ pipette tip and gently washed with PBS. Fresh medium containing 1\% fetal bovine serum was added to the wells with different concentrations of naringenin. At the indicated time after treatment, images were photographed using a microscope (Nikon Eclipse TS100; Nikon Corporation, Tokyo, Japan).

\section{In vitro tube formation assays}

For endothelial cell (EC) tube formation assay, growth factor reduced MatrigelTM (Corning Inc., NY, USA) was thawed overnight at $4{ }^{\circ} \mathrm{C}$. The Matrigel was allowed to solidify on a 24 -well culture plate at $37^{\circ} \mathrm{C}$ for $30 \mathrm{~min}$. HUVECs were harvested and seeded at a density of $1 \times 10^{5}$ cells/well in growth media with or without naringenin. Cells were then incubated at 37 ${ }^{\circ} \mathrm{C}$ for a further $18 \mathrm{~h}$. Tube formation was observed by taking pictures using a microscope (Nikon Corporation). The tube formation assays were quantified by counting the number of tubules from three different fields for each condition. 


\section{Rat aortic ring assays}

For $e x$-vivo angiogenesis study, the rat aortic ring assay was used following a published protocol with modificiations[16]. Briefly, the thoracic aorta from a freshly sacrificed Sprague-Dawley rats (4-week-old, Samtako) was removed in a sterile manner and rinsed in ice cold PBS. It was then cut into $1 \mathrm{~mm}$ long pieces using surgical blade. Each ring was placed in a matrigel pre-coated 24-well-plate. Dulbecco's modified Eagle's medium containing $10 \%$ fetal bovine serum was added to the wells with or without naringenin. Seven days after treatment, the rings were analyzed by microscope (Nikon Corporation) and microvessels sprouting were quantified.

\section{RNA extraction and Reversetranscription (RT)-PCR}

Total RNA was extracted using TRIzol ${ }^{\circledR}$ Reagent (Invitrogen, CA, USA) according to the manufacturer's instructions. The $2 \mu \mathrm{g}$ of total RNA was reverse transcribed into cDNA using SuperScript II Reverse Transcriptase (Invitrogen). The cDNA aliquots were amplified on a Mycyler Thermal Cycler (Bio-Rad) using Go Tag DNA polymerase (Promega) and the gene primers listed in Table 1 . Each PCR cycle consisted of $94{ }^{\circ} \mathrm{C}$ for $1 \mathrm{~min}, 60^{\circ} \mathrm{C}$ for $1 \mathrm{~min}$, and $72{ }^{\circ} \mathrm{C}$ for $1 \mathrm{~min}$, The PCR products were loaded onto a $1.5 \%$ agarose gel containing LoadingSTAR nucleic acid dye (6X, Dynebio, Seongnam, Korea), electrophoresed, and photographed using a Fusion FX7 acquisition system (VilbertLourmat, Eberhardzell, Germany).

Table 1. Primer sequence used for RT-PCR.

\begin{tabular}{lll}
\hline Gene & Primer sequence & Size (bp) \\
\hline Ang2 & 5'-GGATCTGGGGAGAGAGGAAC-3' & 535 \\
& 5'-CTCTGCACCGAGTCATCGTA-3' & \\
Tie2 & 5'-ATCCCATTTGCAAAGCTTCTGGCTGGC-3' & 512 \\
& 5'-TGTGAAGCGTCTCACAGGTCCAGGATG-3' & \\
GAPDH & 5'-ACCACAGTCCATGCCATCAC-3' & 452 \\
& 5'-TCCACCACCCTGTTGCTGTA-3' & \\
\hline
\end{tabular}

\section{Western blot analysis}

At the indicated time, the cells were homogenized in ice-cold lysis buffer containing a protease inhibitor cocktail (Sigma-aldrich). Each protein was separated with SDS-PAGE and transferred to nitrocellulose membranes. After blocking with $5 \%$ skim milk, the membranes were incubated with the following primary antibodies in blocking buffer overnight at $4{ }^{\circ} \mathrm{C}$ : anti-caspase-3 (Cas3) (rabbit), anti-Poly (ADP-ribose) polymerase (PARP), phosphorylated extracellular signalregulated kinases1/2 (p-ERK1/2) (rabbit), andphosphorylated c-Jnk N-terminal kinase ( $\mathrm{p}$-JNK) (mouse) (all from Cell Signaling Technology, Inc.,
Beverly, Ma, USA)and anti- $\beta$-actin (mouse monoclonal; Sigma-Aldrich). Membranes were then incubated with HRP-conjugated secondary antibodies for $1 \mathrm{~h}$ at RT. Chemiluminescent signals were developed with HRP substrate (Millipore) and detected with a Fusion FX7 acquisition system (VilbertLourmat, Eberhardzell, Germany).

\section{Immunocytochemistry}

B16F10 cells and SK-MEL-2 cells cultured on glass coverslips coated with $0.1 \%$ gelatin. Cells were fixed with cold $2 \%$ paraformaldehyde and permeabilized with ice cold $0.5 \%$ Triton X-100 in PBS for $5 \mathrm{~min}$ and blocked in 5\% donkey serum in $0.1 \%$ TritonX-100 in PBS for $1 \mathrm{~h}$ at RT. The cells were incubated with anti-active caspase-3 (rabbit polyclonal; R\&D Systems) overnight at $4{ }^{\circ} \mathrm{C}$. Cells were incubated with Cy3-conjugated donkey anti-rabbit IgG (Jackson ImmunoResearch). Nuclei were stained with 4'6-diamidino-2-phenylindole. Then, the cells were mounted in Fluorescent Mounting Medium (Dako) and immunofluorescent images were acquired using a confocal microscope (Carl Zeiss).

\section{Statistical analysis}

Values are presented as the mean \pm standard deviation (SD). Significant differences between groups were determined by unpaired Student t-tests. For multigroup analysis of variances, one-way or two-way ANOVA was performed followed by Bonferroni post-tests. All statistical analysis was performed using the GraphPad Prism software. Statistical significance was set at $p<0.05$.

\section{Results}

\section{Naringenin treatment inhibits viability and migration of B 16 F 10 murine and SK-MEL-28 human melanoma cells}

B16F10 and SK-MEL-28 melanoma cells were treated for $24 \mathrm{~h}$ in the absence or presence of naringenin $(100,200$, and $400 \mu \mathrm{M})$. Cell viability was determined by a standard MTT assay. The viability results are shown in Fig. 1A, 1B and 1E. Beginning at a concentration of $100 \mu \mathrm{M}$ naringenin, $\mathrm{B} 16 \mathrm{~F} 10$ cell viability progressively decreased from $90.5 \%$ to $43.8 \%$ at $400 \mu \mathrm{M}$ naringenin. Similarly, SK-MEL-28 cell viability was also suppressed with increasing concentration of naringenin, from $78.5 \%$ at $100 \mu \mathrm{M}$ naringenin to $60.9 \%$ at $400 \mu \mathrm{M}$ naringenin. The influence of naringenin on the migration of B16F10 and SK-MEL-28 melanoma cells was also assessed. Confluent cell monolayers were scratched and the subsequent migration of cells in the absence or presence of naringenin $(100,200$, and $400 \mu \mathrm{M})$ was 
observed by optical microscopy. Naringenin at a concentration of 100,200 , and $400 \mu \mathrm{M}$ potently inhibited B16F10 cell migration by approximately $61.1 \%, 88.9 \%$, and $96.7 \%$ versus untreated cells, respectively (Fig. 1C and 1F). Similarly, SK-MEL-28 cell migration was suppressed by 100,200 and $400 \mu \mathrm{M}$ naringenin by $38.3 \%, 43.8 \%$, and $50.8 \%$ versus untreated cells, respectively (Fig. 1D and 1G).

\section{Effect of naringenin treatment on ERK1/2 and JNK signaling pathways}

To investigate the molecular mechanism by which naringenin inhibits melanoma cell viability and migration, B16F10 and SK-MEL-28 cells were cultured and then either not treated with 100, 200, and $400 \mu \mathrm{M}$ naringenin or left untreated. After $18 \mathrm{~h}$ of treatment, in B16F10 cells, naringenin treatment suppressed the protein expression of not only p-ERK1/2 but also $\mathrm{p}$-JNK in a dose-dependent manner as compared to that in untreated B16F10 cells (Fig. 2A and 2B).In

A

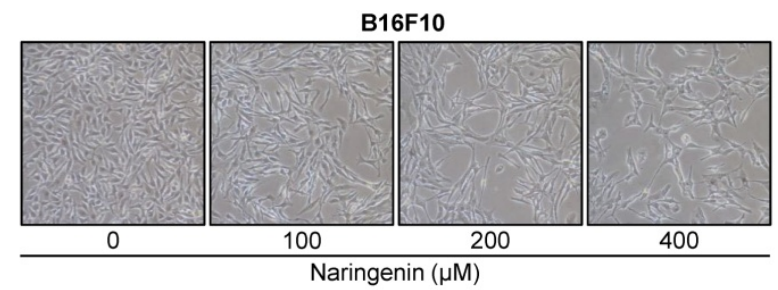

C

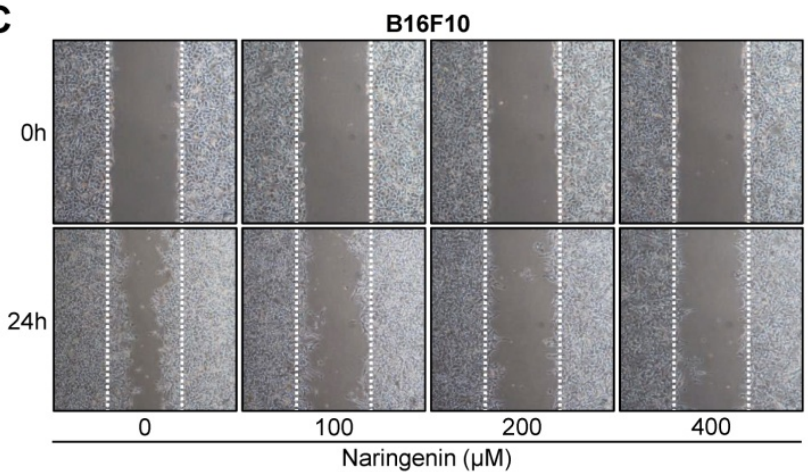

E

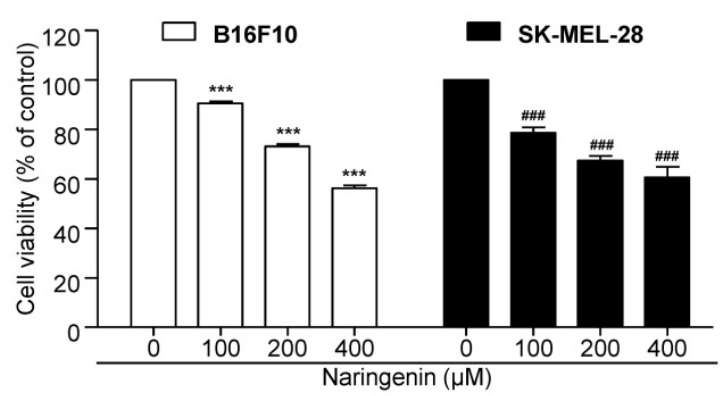

SK-MEL-28 cells, naringenin treatment remarkably inhibited the expression of $\mathrm{p}$-ERK $1 / 2$ protein in a dose-dependent manner whereas it slightly suppressed p-JNK expression (Fig. 2C and 2D).

\section{Effect of naringenin treatment on melanoma cell apoptosis}

To examine the effect of naringenin treatment on induction of cellular apoptosis, Annexin V/Propidium iodide double staining was used. Naringenin treatment induced apoptosis in the B16F10 and SK-MEL-28 melanoma cell lines in a dose-dependent manner (Fig. 3A and 3B). B16F10 cell apoptosis was markedly increased at 100, 200, and 400 $\mu \mathrm{M}$ naringenin more than from two to three times as compared to that of untreated cells. The apoptosis of SK-MEL-28 cells treated with naringenin was gradually increased to approximately two times at 400 $\mu \mathrm{M}$ as compared to that of untreated cells.

B

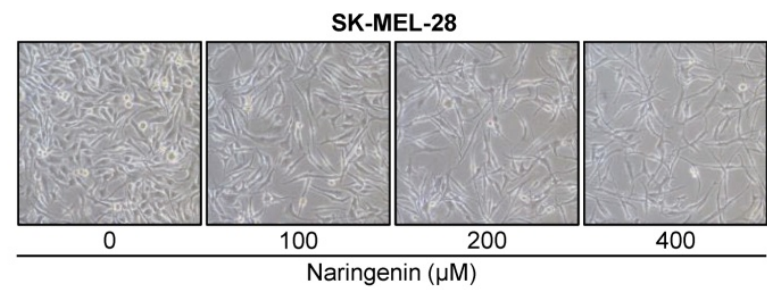

D

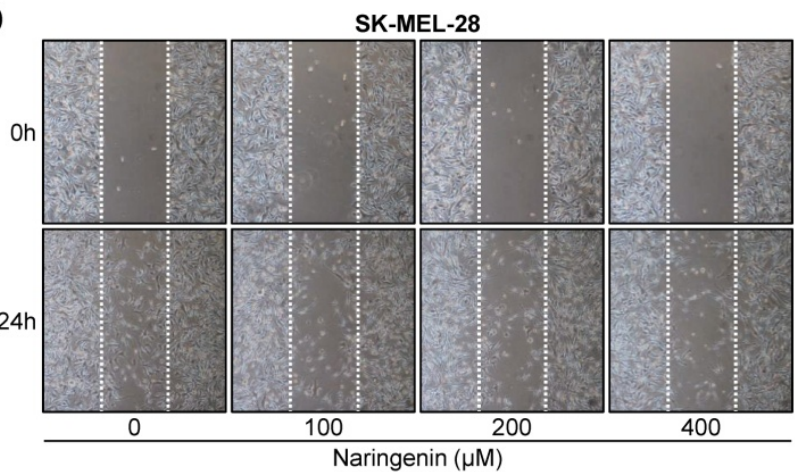

$\mathbf{F}$

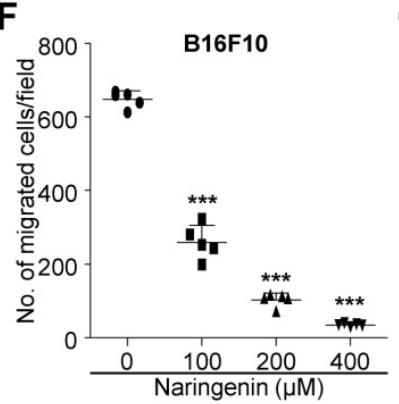

G

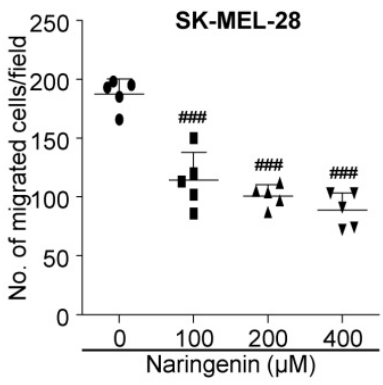

Fig. 1. Inhibitory effect of naringenin treatment on the tumor cell viability and migration. (A, B) Images showing morphological changes on $B 16 \mathrm{~F} 10$ murine (A) and SK-MEL-28 human (B) melanoma cells after naringenin treatment in a dose-dependent manner. Magnification, 100×.(C, D) Images showing B16F10 (C) and SK-MEL-28 (D) cell migration after naringenin treatment. Magnification, 40×. (E) Comparisons of B16F10 and SK-MEL-28 cell viability. (F) Quantification of B16F10 and SK-MEL-28 cell migration after naringenin treatment. Values are mean \pm SD from $\geq$ three independent experiments. $* * * p<0.0001$; \#\#p 0.0001 versus untreated cells by one-way ANOVA followed by Bonferroni post-tests. 
A

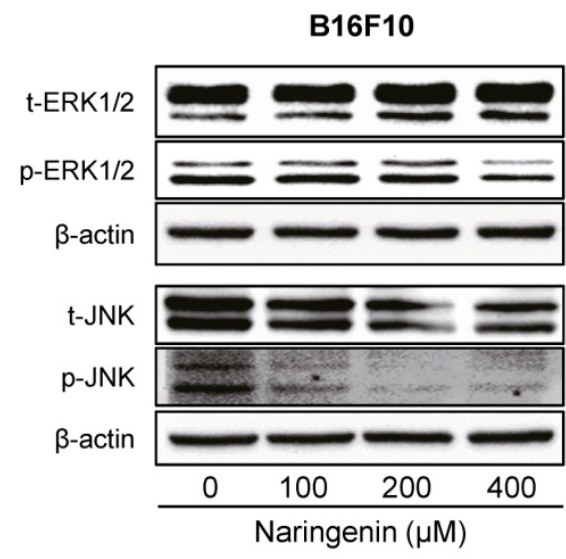

C

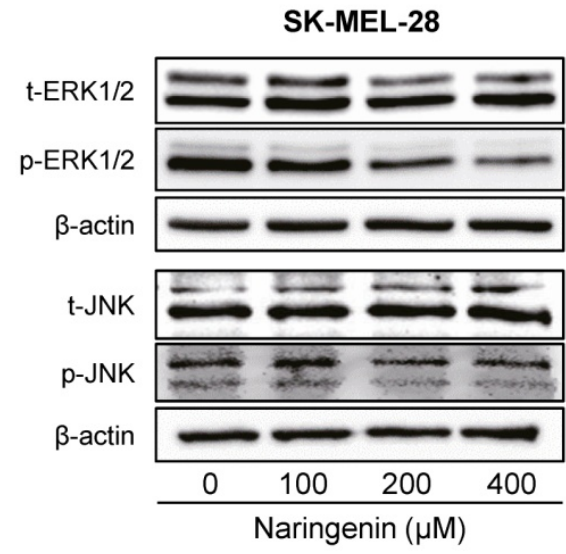

B

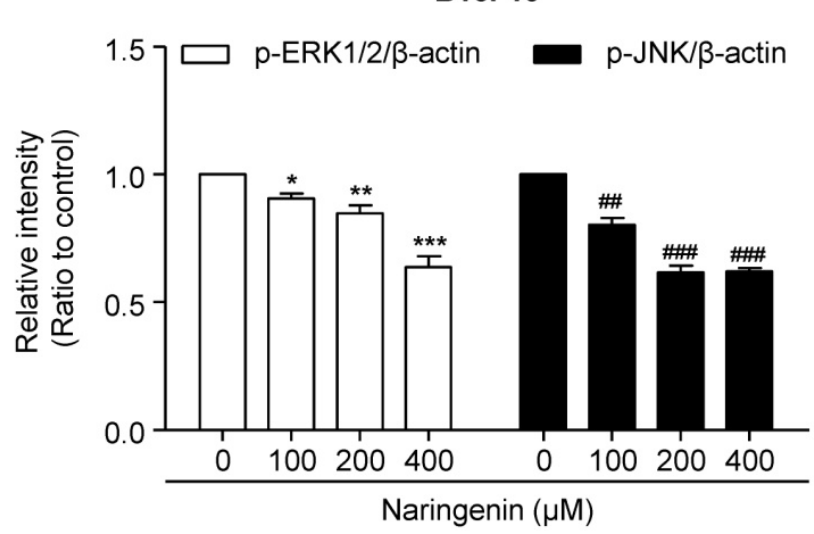

D

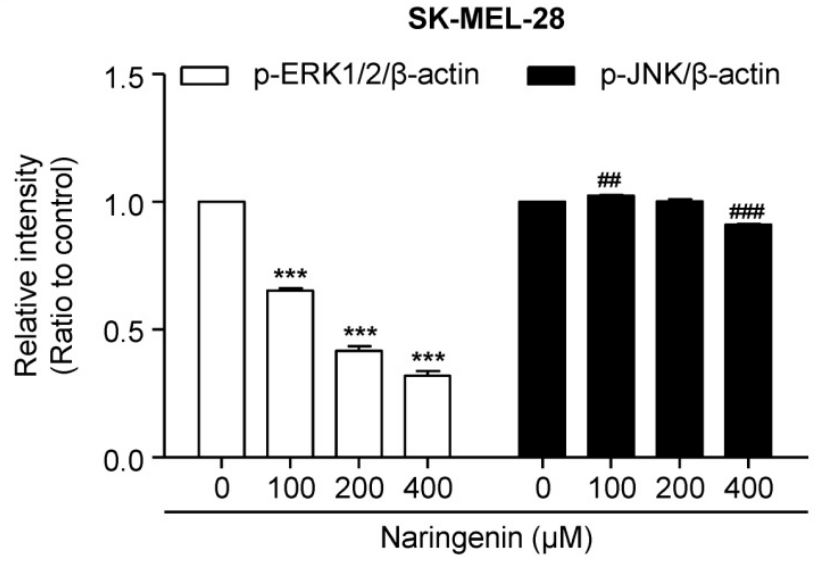

Fig. 2. Effect of naringenin treatment on ERK1/2 and JNK MAPKs signaling. (A-D) Images and quantification of protein expression of $p$-ERK $1 / 2$ and $p-J N K$ in $B 16 F 10$ $(A, B)$ and SK-MEL-28 cells (C, D) after naringenin treatment in a dose-dependent manner. Values are mean \pm SD from $\geq$ three independent experiments. $* p=0.0265$, $* * p<$ $0.0071, * * * p=0.0006 ; \# p=0.0013, \ldots$ \# 0.0002 versus untreated cells by one-way ANOVA followed by Bonferroni post-tests.

To confirm the molecular signaling pathway associated with naringenin-induced apoptosis, we analyzed the expression of pro-apoptotic proteins by western blotting and immunocytochemistry in both the melanoma cell lines. Western blotting revealed the significant increase in activated cas 3 level at $400 \mu \mathrm{M}$ naringenin by approximately two times, as compared to that in untreated B16F10 cells (Fig. 4A and 4B). As PARP acts downstream to cas3, activation of cas 3 by naringenin treatment induced the cleavage of PARP by approximately three times, as compared to that in untreated cells. Similarly, in SK-MEL-28 cells, $400 \mu \mathrm{M}$ naringenin treatment significantly increased the intensity of active cas 3 and active PARP signal by approximately one and a half times as compared to that in untreated cells (Fig. 4C and 4D).For Immunocytochemistry, we used a $400 \mu \mathrm{M}$ concentration where induced cell apoptosis. Immunochemical data revealed naringenin activates the cas3 in B16F10 and SK-MEL-28, supporting above western blotting results (Fig. 4E and 4F).

\section{Naringenin exerts anti-angiogenic effects by inhibiting EC migration, tube formation, and microvessel sprouting}

To assess the inhibitory effects of naringenin on EC migration, HUVECs were seeded and allowed to grow to a confluent monolayer. The monolayer was scratched and then either untreated or treated with 10, 100 , and $200 \mu \mathrm{M}$ naringenin. After $12 \mathrm{~h}$ and $24 \mathrm{~h}$, the cell migration was observed by optical microscopy. The representative photographs and their quantification showed the migration of HUVECs was markedly decreased following treatment with 100 and $200 \mu \mathrm{M}$ naringenin (Fig. 5A and 5B). We used a 200 $\mu \mathrm{M}$ concentration for subsequent angiogenesis experiments. Since cell migration is crucial in tube formation of HUVECs [17], we suggest that naringenin may inhibit the tube formation of HUVECs as well as the cell migration. HUVECs were seeded on Matrigel with or without naringenin treatment and incubated for $18 \mathrm{~h}$. The number of 
tubules was determined. Naringenin remarkably inhibited tube formation of HUVECs in a dose-dependent manner (Fig. 5C and 5D, Supplementary Fig. $1 \mathrm{~A}$ and $1 \mathrm{~B}$ ). Furthermore, to assess the inhibitory effect of naringenin on microvessel sprouting, an ex vivo rat aorta ring assay was used. Treatment with $200 \mu \mathrm{M}$ naringenin significantly attenuated the sprouting of microvessels from rat aorta ring in a dose-dependent manner (Fig. 5E and 5F, Supplementary Fig. 1C and 1D).

\section{Effects of naringenin treatment on expression of Ang2/Tie2.}

Since the Angiopoietin-2 (Ang2)/Tyrosine- protein kinase receptor-2 (Tie2) kinase signaling pathway is pivotal in tumor angiogenesis [18], we investigated the influence of naringenin treatment on this pathway. HUVECs were seeded and were either treated with $200 \mu \mathrm{M}$ naringenin for $12 \mathrm{~h}$ and $24 \mathrm{~h}$ or left untreated. At each time, the mRNA expression of Ang2/Tie2 was confirmed by RT-PCR. Naringenin treatment remarkably reduced mRNA expression of the Tie2, as compared to that in untreated cells (Fig. $6 \mathrm{~A}$ and $6 \mathrm{~B})$. However, naringenin treatment slightly affected the mRNA expression of Ang2 (Fig. 6C and $6 \mathrm{D})$.
A

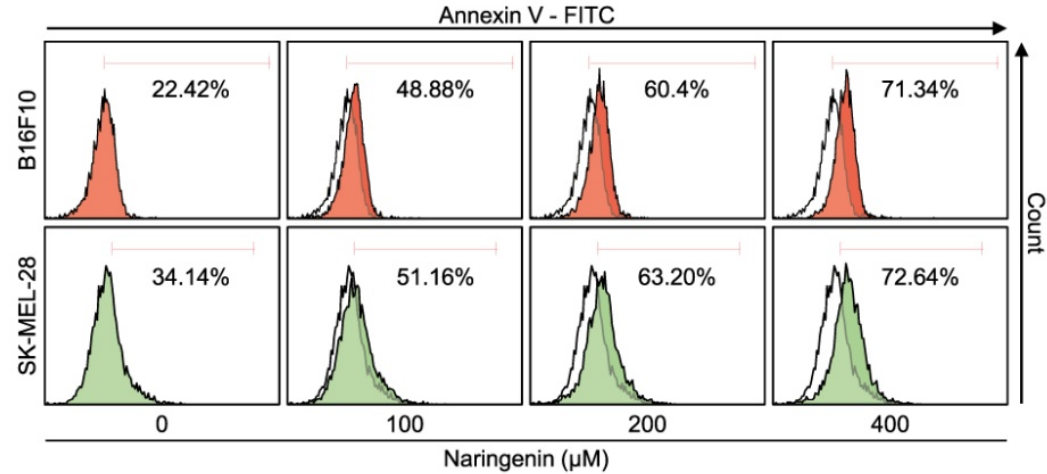

B

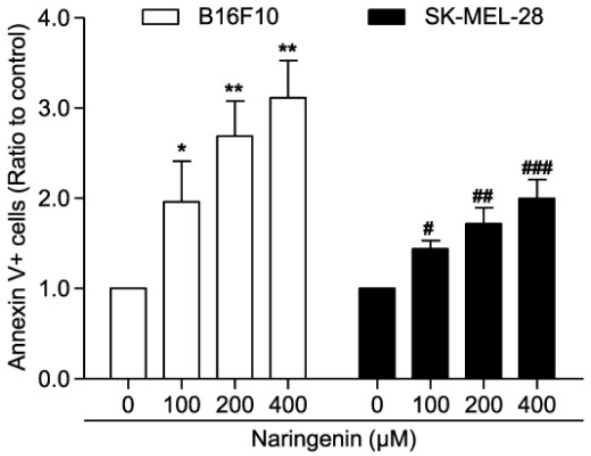

Fig. 3. Naringenin treatment induces cell apoptosis. (A, B) Flow cytometry analysis and quantification showing apoptosis in B16F10 and SK-MEL-28 cells. Values are

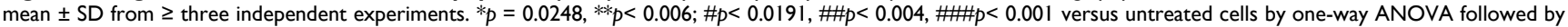
Bonferroni post-tests.

A

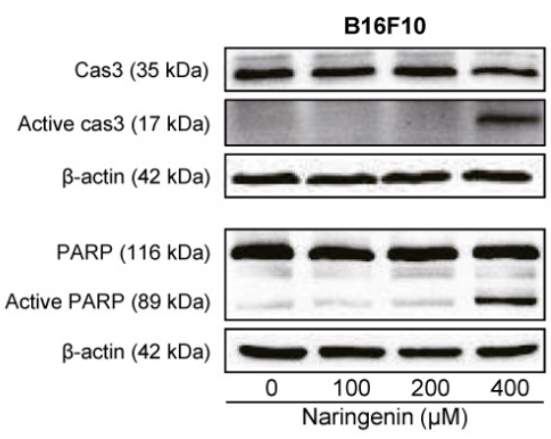

C

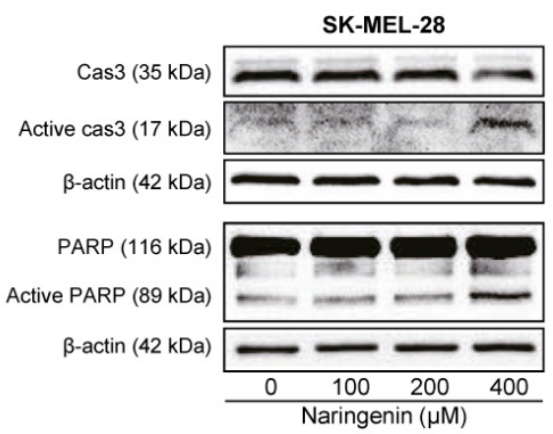

B

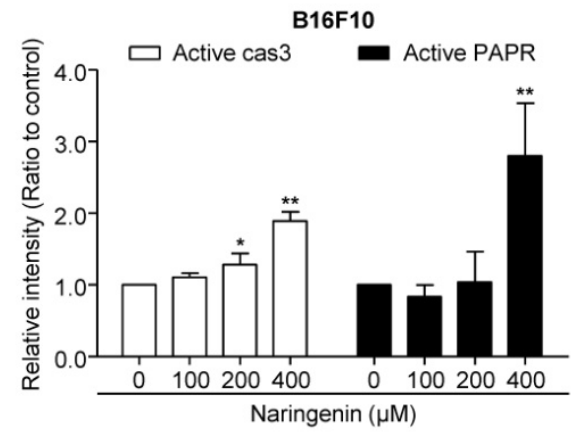

D

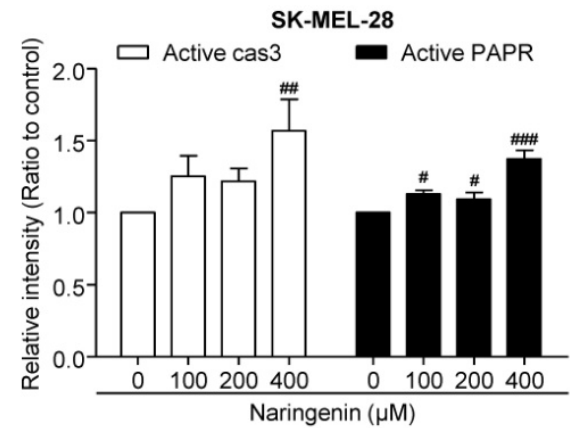

$\mathbf{E}$

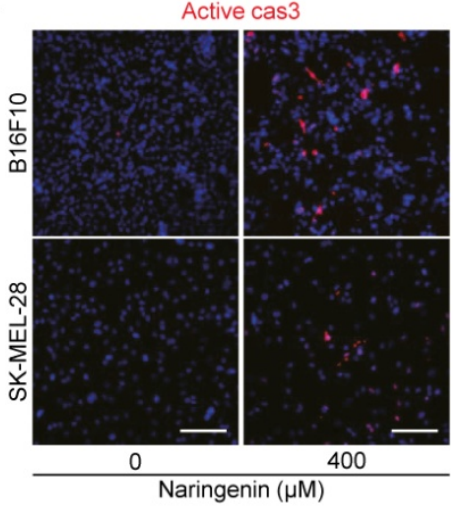

$\mathbf{F}$

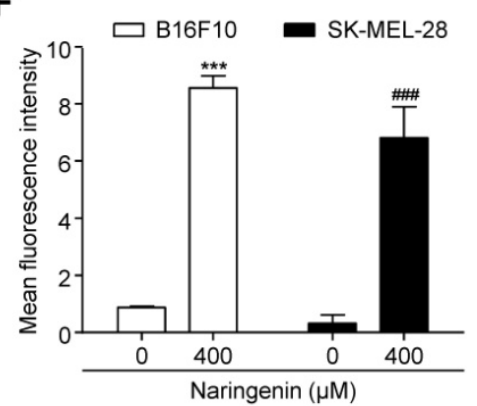

Fig. 4. Naringenin treatment leads to activation of cas 3 and PARP. (A, B)Images and quantification of active cas $3^{+}$area in B16F10 and SK-MEL-28 cells.Scale bar, 100 $\mu \mathrm{m}$. (C-F)Images and quantification of protein expression of cas3, active cas3 and PARP, active PARP in B16F10 (C, D) and SK-MEL-28 cells (E, F) after naringenin treatment in a dose-dependent manner. Values are mean \pm SD from $\geq$ three independent experiments. ${ }^{*} p=0.0340,{ }^{* *} p=0.0013, * * * p<0.0001 ; \# p<0.05, \# \# p=0.01, \# \# p<0.001$ versus untreated cells by one-way ANOVA followed by Bonferroni post-tests. 
A

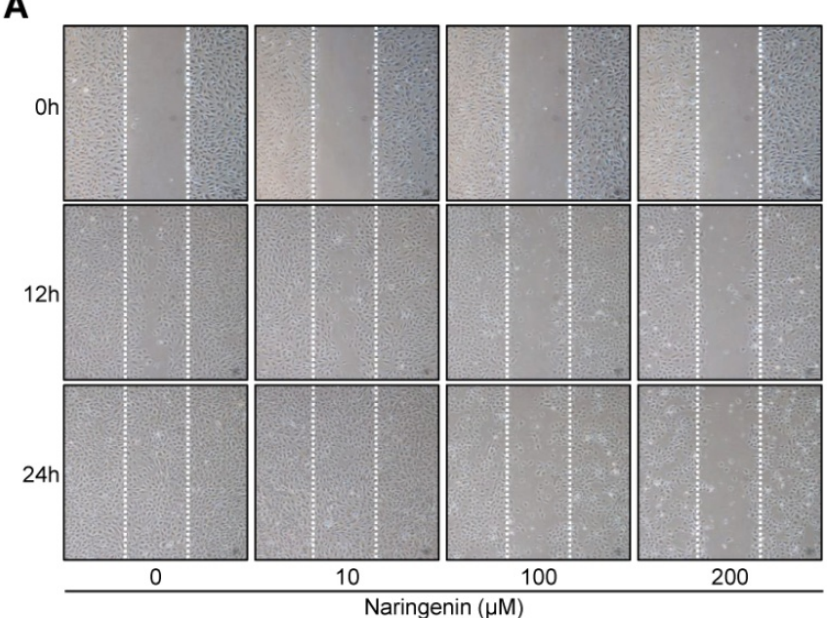

B

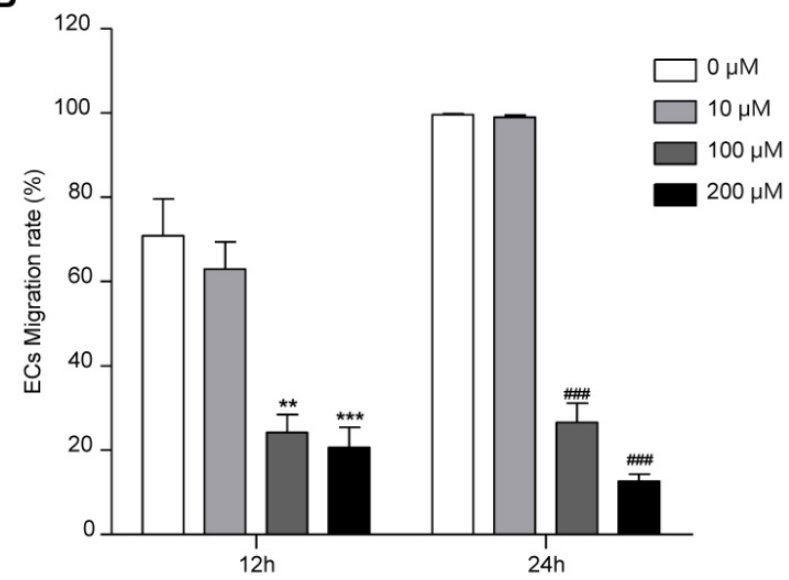

E

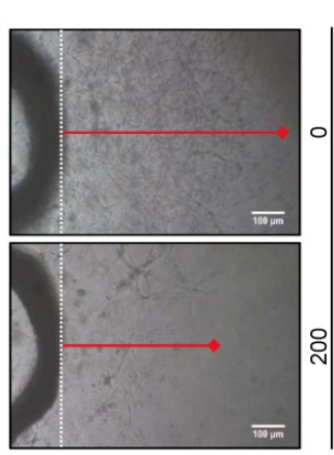

$\mathbf{F}$

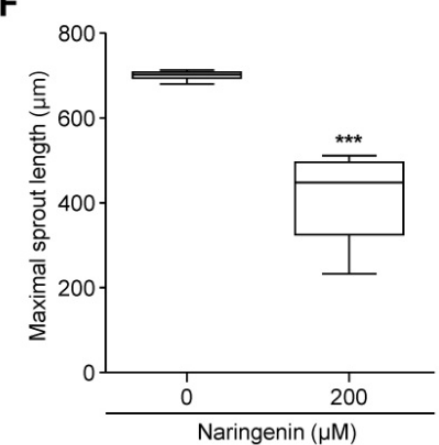

Fig. 5. Naringenin treatment inhibits angiogenesis in vitro and ex vivo. (A, B) Images showing HUVECs migration and comparisons of ECs migration rate. Data are presented as a percentage of scratched area at time $0 \mathrm{~h}$. Magnification, $40 \times$. Values are mean \pm SD from $\geq$ three independent experiments. $* * p=0.0013$, $* * * p=0.0003$; \#\#\# $<$ 0.001 versus untreated group by two-way ANOVA followed by Bonferroni post-tests. (C, D) Images showing ECs tube formation and comparisons of number of tubules. Magnification, 100×.(E, F) Images showing sprouting of ECs from rat aorta and comparisons of maximal length of ECs sprouting.Magnification, $40 \times$. Values are mean \pm SD from $\geq$ three independent experiments. $* * * p<0.0001$ versus untreated group by unpaired t-test.

A

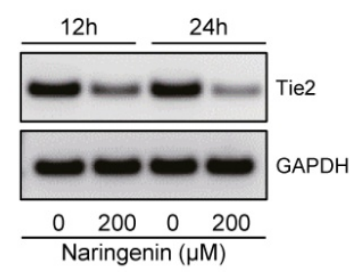

B

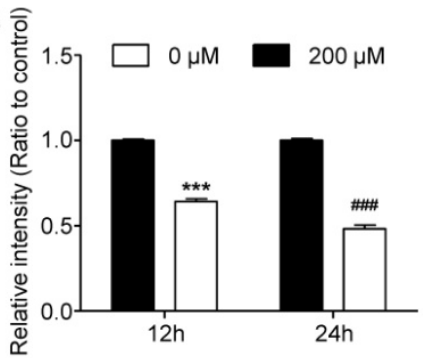

C

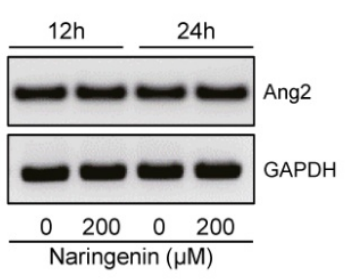

D 흐

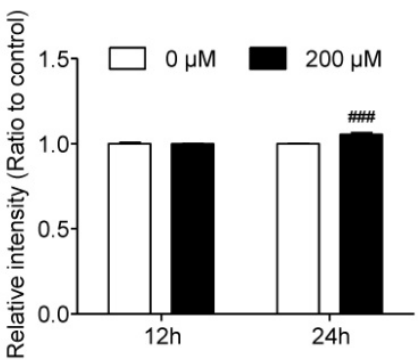

Fig. 6. Effect of naringenin treatment on expression of Ang2 and Tie2 mRNA in HUVECs. (A, B) Images and quantification of mRNA expression Tie2 in HUVECs after naringenin treatment. (C, D) Images and quantification of mRNA expression Ang2 in HUVECs after naringenin treatment. Values are mean \pm SD from $\geq$ three independent experiments. $* * * p<0.0001, \ldots \# p 0.0006$ versus untreated group by unpaired t-test.

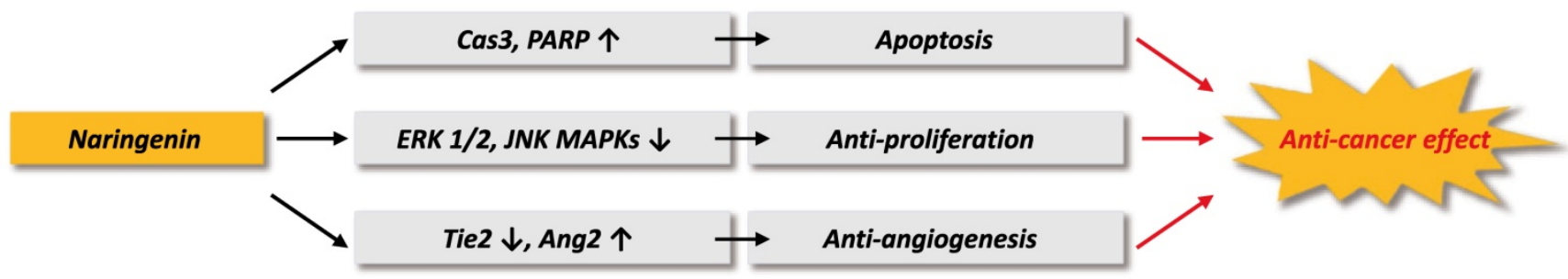

Fig. 7. Schematic diagram of anti-cancer effect of naringenin.

\section{Discussion}

The documented therapeutic activities of naringenin, a major flavonoid, include anti-inflammatory [19], anti-diabetic [20], and anti-oxidative [21] activities. Particularly, naringenin exerts anticancer effects on various cancer cells [22-24]. In the present study, we investigated whether 
naringenin also has anticancer effects against B16F10 murine and SK-MEL-28 human melanoma cells. We also assessed the effects of naringenin treatment on angiogenesis of HUVECs and ex vivo sprouting of microvessels.

Cell proliferation and migration of tumor cells are necessary processes during tumor progression. We observed that naringenin inhibited cell proliferation and migration in a dose-dependent manner in B16F10 and SK-MEL-28 cells. We further examined the phosphorylation status of Mitogen-activated protein kinases (MAPKs) by immunoblotting to elucidate the molecular mechanisms. MAPK signaling pathways, including ERK1/2 and JNK, are involved in survival and proliferation [25]. Overexpression of phosphorylated MAPKs has been found in various cancer cell lines [26]. In this study, we confirmed that naringenin suppressed the B16F10 and SK-MEL-28 cell growth by inhibiting the phosphorylation of ERK1/2 and JNK MAPKs. MAPK signaling pathway is initiated by activation of receptors including RTK in response to various extracellular signals such as growth factors, followed by activating a cascade signaling events of three kinases (i.e., MAPKKK-MAPKK-MAPK)[27]. Therefore, it may be suggested naringenin suppressed the activation of ERK1/2 and JNK MAPKs by targeting upstream proteins involved in the cascade of MAPK signaling.

The anti-cancer effect of naringenin on B16F10 melanoma cell line has been previously observed. Iwashita et al. revealed the growth inhibitory activity of naringenin on B16 mouse melanoma 4 A5 cells [28]. Bouzaiene et al. demonstrated that naringenin significantly suppressed the cell proliferation and induced the cell apoptosis on B16F10 cells [29]. Similar to previous studies, we observed anti-proliferative activities of naringenin on B16F10 cells in a dose-dependent manner. However, our study differs from previous studies in two ways. First, we used human melanoma SK-MEL-28 cells. Second, we revealed the mechanisms underlying naringenininduced cell apoptosis using protein expression analysis. In our studies, naringenin induced B16F10 and SK-MEL-28 cell death in a dose-dependent manner. Apoptosis is the most popular underlying mechanism of the anticancer effects of various anticancer drugs, including natural compounds [30].Similar to previous reports [31,32], we found that naringenin killed tumor cells by inducing apoptosis. Furthermore, naringenin treatment significantly upregulated the expression of activated cas3 and PARP at $400 \mu \mathrm{M}$. Cas 3 activate cleavage of PARP, and so is crucial in apoptosis. The present findings demonstrate that the increased activation of cas 3 by naringenin treatment simultaneously induced PARP activation. These results indicate that naringenin induces apoptosis in a cas3-dependent manner.

Tumor angiogenesis is recognized as a pivotal cancer hallmark that is essential for tumor progression [33]. Targeting tumor angiogenesis has been viewed as a promising strategy for clinical cancer therapy. However, clinical trials indicated that traditional anti-angiogenic treatment is limited and may instead accelerate tumorigenesis. In the present study, the results showed that naringenin treatment significantly reduced the migration of HUVECs in a dose-dependent manner. Moreover, the results of an in vitro EC tube formation assay on Matrigel and an $e x$ vivo rat aorta ring assay demonstrated that naringenin treatment potently suppressed tube formation of HUVECs and sprouting of microvessels. Further study to understand the underlying mechanisms of the anti-angiogenic effects of naringenin, revealed that naringenin treatment significantly reduced the gene expression of Tie-2, but did not inhibit the expression of Ang2. These results suggest that the anti-angiogenesis effects of naringenin involve the downregulation of Tie2 expression.

Pafumi et al. demonstrated the anti-angiogenic effect of naringenin in vitro and in vivo[34]. Similar to our results, they showed that naringenin impaired not only VEGF-induced vessel formation but also neovascularization in C57BL/6 mice. However, our study is different from theirs in that we additionally investigated the inhibitory effect on HUVECs migration. Furthermore, to provide potential molecular mechanisms of the anti-angiogenic effects of naringenin, we identified the expression of angiogenic factors (i.e. Ang2, Tie2). However, they suggested that naringenin inhibits VEGF-induced angiogenesis by impaired intracellular calcium signaling.

In conclusion, we demonstrated the anticancer effect of naringenin and its potential mechanisms, as illustrated in Figure 7 . Naringenin treatment suppressed proliferation and migration of B16F10 murine and SK-MEL-28 human melanoma cell lines by inhibiting the phosphorylation of ERK1/2 and JNK MAPKs. Naringenin treatment also induced cas3-dependent apoptosis in the melanoma cells, suppressed HUVECs migration and tube formation, and inhibited ex vivomicrovessel sprouting by regulating the mRNA expression of Tie-2 and Ang-2. The collective results demonstrate the anticancer effects of naringenin via its induction of tumor cell death and inhibition of angiogenesis in malignant melanoma. Naringenin has potential as an effective and safe therapeutic agent for malignant melanoma. 


\section{Supplementary Material}

Supplementary figure.

http://www.medsci.org/v17p3049s1.pdf

\section{Acknowledgments}

This work was supported by Korea Institute of Planning and Evaluation for Technology in Food, Agriculture, Forestry (IPET) through Agriculture, Food and Rural Affairs Research Center Support Program, funded by Ministry of Agriculture, Food and Rural Affairs (MAFRA; 716002-7).

\section{Competing Interests}

The authors have declared that no competing interest exists.

\section{References}

1. Khazaei Z, Ghorat F, Jarrahi A, Adineh H, Sohrabivafa M, Goodarzi E. Global Incidence And Mortality Of Skin Cancer By Histological Subtype And Its Relationship With The Human Development Index (Hdi); An Ecology Study In 2018. World Cancer Research Journal. 2019; 6: 13

2. Ward WH, Farma JM. The Epidemiology of Cutaneous Melanoma in the White and Black African Population Groups in South Africa--Cutaneous Melanoma: Etiology and Therapy: Codon Publications; 2017.

3. Bhatia S, Tykodi SS, Thompson JA. Treatment of metastatic melanoma: an overview. Oncology (Williston Park, NY). 2009; 23: 488.

4. Shapiro CL. Highlights of Recent Findings on Quality-of-Life Management for Patients With Cancer and Their Survivors. JAMA oncology. 2016; 2: 1401-2.

5. Abotaleb M, Samuel SM, Varghese E, Varghese S, Kubatka P, Liskova A, et al. Flavonoids in cancer and apoptosis. Cancers. 2019; 11: 28.

6. Wang S, Shen P, Zhou J, Lu Y. Diet phytochemicals and cutaneous carcinoma chemoprevention: A review. Pharmacological research. 2017; 119: 327-46.

7. El-Rahman SSA, Shehab G, Nashaat H. Epigallocatechin-3-Gallate: The prospective targeting of cancer stem cells and preventing metastasis of chemically-induced mammary cancer in rats. The American journal of the medical sciences. 2017; 354: 54-63.

8. He Z, Chen AY, Rojanasakul Y, Rankin GO, Chen YC. Gallic acid, a phenolic compound, exerts anti-angiogenic effects via the PTEN/AKT/HIF-1a/VEGF signaling pathway in ovarian cancer cells. Oncology reports. 2016; 35: 291-7.

9. Jin G, Yang Y, Liu K, Zhao J, Chen X, Liu H, et al. Combination curcumin and (-)-epigallocatechin-3-gallate inhibits colorectal carcinoma microenvironment-induced angiogenesis by JAK/STAT3/IL-8 pathway. Oncogenesis. 2017; 6: e384-e.

10. Salehi B, Fokou PVT, Sharifi-Rad M, Zucca P, Pezzani R, Martins N, et al. The Therapeutic Potential of Naringenin: A Review of Clinical Trials. Pharmaceuticals (Basel). 2019; 12: 11.

11. Chandrika BB, Steephan M, Kumar TRS, Sabu A, Haridas M. Hesperetin and Naringenin sensitize HER2 positive cancer cells to death by serving as HER2 Tyrosine Kinase inhibitors. Life Sciences. 2016; 160: 47-56.

12. Hernández-Aquino E, Muriel P. Beneficial effects of naringenin in liver diseases: Molecular mechanisms. World journal of gastroenterology. 2018; 24 : 1679.

13. Ke JY, Banh T, Hsiao YH, Cole RM, Straka SR, Yee LD, et al. Citrus flavonoid naringenin reduces mammary tumor cell viability, adipose mass, and adipose inflammation in obese ovariectomized mice. Mol Nutr Food Res. 2017; 61.

14. Lim W, Park S, Bazer FW, Song G. Naringenin-Induced Apoptotic Cell Death in Prostate Cancer Cells Is Mediated via the PI3K/AKT and MAPK Signaling Pathways. J Cell Biochem. 2017; 118: 1118-31.

15. Li Q, Wang Y, Zhang L, Chen L, Du Y, Ye T, et al. Naringenin exerts anti-angiogenic effects in human endothelial cells: Involvement of ERRa/VEGF/KDR signaling pathway. Fitoterapia. 2016; 111: 78-86.

16. Saraswati S, Agrawal SS. Brucine, an indole alkaloid from Strychnos nux-vomica attenuates VEGF-induced angiogenesis via inhibiting VEGFR2 signaling pathway in vitro and in vivo. Cancer Letters. 2013; 332: 83-93.

17. Zang M, Hu L, Zhang B, Zhu Z, Li J, Zhu Z, et al. Luteolin suppresses angiogenesis and vasculogenic mimicry formation through inhibiting Notch1-VEGF signaling in gastric cancer. Biochemical and biophysical research communications. 2017; 490: 913-9.

18. Rigamonti N, Kadioglu E, Keklikoglou I, Wyser Rmili C, Leow CC, De Palma M. Role of angiopoietin-2 in adaptive tumor resistance to VEGF signaling blockade. Cell reports. 2014; 8: 696-706.

19. Xue N, Wu X, Wu L, Li L, Wang F. Antinociceptive and anti-inflammatory effect of Naringenin in different nociceptive and inflammatory mice models. Life sciences. 2019; 217: 148-54.
20. Den Hartogh DJ, Tsiani E. Antidiabetic properties of naringenin: A citrus fruit polyphenol. Biomolecules. 2019; 9: 99.

21. Zaidun $\mathrm{NH}$, Thent ZC, Latiff AA. Combating oxidative stress disorders with citrus flavonoid: Naringenin. Life sciences. 2018; 208: 111-22.

22. Zhang H, Zhong X, Zhang X, Shang D, Zhou Y, Zhang C. Enhanced anticancer effect of ABT-737 in combination with naringenin on gastric cancer cells. Experimental and therapeutic medicine. 2016; 11: 669-73.

23. Wang $R$, Wang J, Dong $T$, Shen J, Gao X, Zhou J. Naringenin has a chemoprotective effect in MDA-MB-231 breast cancer cells via inhibition of caspase-3 and-9 activities. Oncology letters. 2019; 17: 1217-22.

24. Chang H-L, Chang Y-M, Lai S-C, Chen K-M, Wang K-C, Chiu T-T, et al. Naringenin inhibits migration of lung cancer cells via the inhibition of matrix metalloproteinases-2 and -9. Experimental and therapeutic medicine. 2017; 13: 739-44.

25. Raman M, Chen W, Cobb M. Differential regulation and properties of MAPKs. Oncogene. 2007; 26: 3100-12.

26. Burotto M, Chiou VL, Lee J-M, Kohn EC. The MAPK pathway across different malignancies: a new perspective. Cancer. 2014; 120: 3446-56.

27. Papa S, Choy PM, Bubici C. The ERK and JNK pathways in the regulation of metabolic reprogramming. Oncogene. 2019; 38: 2223-40.

28. Iwashita $\mathrm{K}$, Kobori M, Yamaki $\mathrm{K}$, Tsushida T. Flavonoids inhibit cell growth and induce apoptosis in B16 melanoma 4A5 cells. Biosci Biotechnol Biochem. 2000; 64: 1813-20.

29. Nasr Bouzaiene N, Chaabane F, Sassi A, Chekir-Ghedira L, Ghedira K. Effect of apigenin-7-glucoside, genkwanin and naringenin on tyrosinase activity and melanin synthesis in B16F10 melanoma cells. Life Sci. 2016; 144: 80-5.

30. Shi Y. Caspase activation: revisiting the induced proximity model. Cell. 2004; 117: 855-8.

31. Zhao Z, Jin G, Ge Y, Guo Z. Naringenin inhibits migration of breast cancer cells via inflammatory and apoptosis cell signaling pathways. Inflammopharmacology. 2019; 27: 1021-36.

32. Park HJ, Choi YJ, Lee JH, Nam MJ. Naringenin causes ASK1-induced apoptosis via reactive oxygen species in human pancreatic cancer cells. Food and chemical toxicology : an international journal published for the British Industrial Biological Research Association. 2017; 99: 1-8.

33. Hu Z, Brooks SA, Dormoy V, Hsu C-W, Hsu H-Y, Lin L-T, et al. Assessing the carcinogenic potential of low-dose exposures to chemical mixtures in the environment: focus on the cancer hallmark of tumor angiogenesis. Carcinogenesis. 2015; 36: S184-S202.

34. Pafumi I, Festa M, Papacci F, Lagostena L, Giunta C, Gutla V, et al. Naringenin Impairs Two-Pore Channel 2 Activity And Inhibits VEGF-Induced Angiogenesis. Sci Rep. 2017; 7: 5121. 